\title{
Minimizing Regret with Multiple Reserves
}

\author{
TIM ROUGHGARDEN and JOSHUA R. WANG, Stanford University
}

We study the problem of computing and learning non-anonymous reserve prices to maximize revenue. We first define the MAXIMIZING Multiple Reserves (MMR) problem in single-parameter matroid environments, where the input is $m$ valuation profiles $\mathbf{v}^{1}, \ldots, \mathbf{v}^{m}$, indexed by the same $n$ bidders, and the goal is to compute the vector $\mathbf{r}$ of (non-anonymous) reserve prices that maximizes the total revenue obtained on these profiles by the VCG mechanism with reserves $\mathbf{r}$. We prove that the problem is $A P X$-hard, even in the special case of single-item environments, and give a polynomial-time $\frac{1}{2}$-approximation algorithm for it in arbitrary matroid environments.

We then consider the online no-regret learning problem and show how to exploit the special structure of the MMR problem to translate our offline approximation algorithm into an online learning algorithm that achieves asymptotically time-averaged revenue at least $\frac{1}{2}$ times that of the best fixed reserve prices in hindsight. On the negative side, we show that, quite generally, computational hardness for the offline optimization problem translates to computational hardness for obtaining vanishing time-averaged regret. Thus, our hardness result for the MMR problem implies that computationally efficient online learning requires approximation, even in the special case of single-item auction environments.

CCS Concepts: • Theory of computation $\rightarrow$ Computational pricing and auctions; Approximation algorithms analysis; Online algorithms; Algorithmic mechanism design;

Additional Key Words and Phrases: Second-price auction, non-anonymous reserve prices, no alpha-regret

\section{ACM Reference format:}

Tim Roughgarden and Joshua R. Wang. 2019. Minimizing Regret with Multiple Reserves. ACM Trans. Econ. Comput. 7, 3, Article 17 (October 2019), 18 pages.

https://doi.org/10.1145/3355900

\section{INTRODUCTION}

A basic issue in the design and deployment of revenue-maximizing auctions is the determination of appropriate reserve prices. For example, consider the well-studied problem of selecting an anonymous reserve price in a second-price (Vickrey) auction for a single item-equivalently, an opening bid in an eBay auction. ${ }^{1}$ There are several versions of the problem, depending on the informational

\footnotetext{
${ }^{1}$ In a second-price single-item auction with a reserve price $r$, the winner is the highest bidder that clears the reserve (if any), and the selling price is the maximum of $r$ and the second-highest bid. Bidding truthfully is a dominant strategy for every bidder.

A preliminary version of this work appeared in the EC 2016 conference (Roughgarden and Wang 2016).

This research was supported in part by NSF grants CCF-1215965 and CCF-1524062, and a Stanford Graduate Fellowship. Authors' addresses: T. Roughgarden, Department of Computer Science, Stanford University, 474 Gates Building, 353 Serra Mall, Stanford, CA 94305; email: tim@cs.stanford.edu; J. R. Wang, Department of Computer Science, Stanford University, 460 Gates Building, 353 Serra Mall, Stanford, CA 94305; email: joshua.wang@cs.stanford.edu.

Permission to make digital or hard copies of all or part of this work for personal or classroom use is granted without fee provided that copies are not made or distributed for profit or commercial advantage and that copies bear this notice and the full citation on the first page. Copyrights for components of this work owned by others than ACM must be honored. Abstracting with credit is permitted. To copy otherwise, or republish, to post on servers or to redistribute to lists, requires prior specific permission and/or a fee. Request permissions from permissions@acm.org.

(C) 2019 Association for Computing Machinery.

2167-8375/2019/10-ART17 \$15.00

https://doi.org/10.1145/3355900
} 
assumptions. For context, we next review four different setups; this article is primarily about the second and fourth ones (in the more complicated setting of non-anonymous reserve prices).

Bayesian optimization. The standard economic approach is to assume a prior distribution over the valuations of the bidders participating in the auction, and to maximize expected revenue with respect to this distribution. If bidders' valuations are drawn i.i.d. from a distribution that satisfies some modest technical conditions ("regularity"), then setting an anonymous reserve price equal to the distribution's monopoly price yields an optimal auction (Myerson 1981). Chawla et al. (2007), Hartline and Roughgarden (2009), and Alaei et al. (2015) provide guidance on how to set the reserve price when the i.i.d. or regularity assumptions are relaxed.

Offline optimization. Given $m$ arbitrary valuation profiles $\mathbf{v}^{1}, \ldots, \mathbf{v}^{m}$ (each an $n$-vector, where $n$ is the number of bidders), the goal here is to determine the anonymous reserve price of a second-price auction that maximizes the average auction revenue across these profiles. $^{2}$ There is always an optimal reserve price equal to some bidder's valuation in one of the $m$ profiles, so there are at most $m n$ different reserve prices that need to be tried. Thus, the problem is easy to solve in polynomial time.

Batch learning. In this version, there is an unknown distribution $F$ over bidders' valuations. A learning algorithm is given $m$ i.i.d. samples from $F$, and must then output a reserve price. The goal is to output a reserve price that achieves expected revenue (w.r.t. fresh draws from $F$ ) close to that obtained by the best reserve price (for $F$ ), with high probability over the samples. This problem was studied implicitly by Dhangwatnotai et al. (2015) and explicitly by Medina and Mohri (2014) and Huang et al. (2018).

Online vanishing-regret learning. In online learning, valuation profiles $\mathbf{v}^{1}, \ldots, \mathbf{v}^{T}$ arrive one at a time, and at time $t$ a learning algorithm chooses a reserve price as a function of the previously seen profiles $\mathbf{v}^{1}, \ldots, \mathbf{v}^{t-1}$. The goal is to choose reserve prices over time so the time-averaged revenue is almost as high as that achieved by the best fixed reserve price in hindsight. Versions of this problem were studied by Kleinberg and Leighton (2003), Blum and Hartline (2005), and Cesa-Bianchi et al. (2013). ${ }^{3}$

There are well-known connections between these different problems. For example, an efficient exact or approximate algorithm for the offline optimization problem implies, in "black-box" fashion, an efficient learning algorithm for the batch learning problem with the same approximation factor (namely, the "empirical risk minimizer" (ERM); see, e.g., Anthony and Bartlett (1999)). An efficient exact algorithm for the offline problem can also be translated into an efficient online vanishingregret learning algorithm (see, e.g., Cesa-Bianchi and Lugosi (2006)). ${ }^{4}$

In this article, we study the problem of computing or learning non-anonymous reserve prices, where a different reserve price $r_{i}$ can be used for each bidder $i$. For example, in a single-item second-price auction with reserves $r_{1}, \ldots, r_{n}$, the winner is the highest bidder who clears her

\footnotetext{
${ }^{2} \mathrm{An}$ alternative interpretation of the problem is of maximizing expected revenue with respect to a correlated distribution over valuation profiles (namely, the uniform distribution over $\mathbf{v}^{1}, \ldots, \mathbf{v}^{m}$ ).

${ }^{3}$ In fact, there are two well-studied versions of vanishing-regret online learning: the stochastic case (where every valuation profile is drawn i.i.d. from an unknown distribution) and the adversarial case (where each valuation profile is arbitrary). This article studies only the second, more difficult, setting.

${ }^{4}$ The positive results by Kleinberg and Leighton (2003) and Cesa-Bianchi et al. (2013) are interesting and non-trivial, because they apply even when the price-setter receives only limited feedback from each auction.
} 
reserve (if any), and the selling price is either her reserve price or the second-highest bid of a bidder who cleared her own reserve price, whichever is larger. ${ }^{5}$

All four genres of problems are relevant and interesting with non-anonymous reserve prices; we study the latter three in this work.

Bayesian optimization. Hartline and Roughgarden (2009), extending Chawla et al. (2007), study the power of non-anonymous reserve prices in Bayesian settings, where bidders' valuations are independently but not identically distributed. The main results show that setting each bidder's reserve to the monopoly price for her distribution yields a mechanism with near-optimal expected revenue in many scenarios. ${ }^{6}$

Offline optimization. Given $m$ arbitrary valuation profiles $\mathbf{v}^{1}, \ldots, \mathbf{v}^{m}$, each indexed by the same set of bidders, the goal is now to compute one reserve price per bidder to maximize the average revenue of the second-price auction with reserves across these profiles. ${ }^{7}$ In contrast to the anonymous reserve price setting, this optimization problem is non-trivial: There are now $m^{n}$ different price vectors that could be optimal. This problem has not been studied previously, except for the independent work by Leme et al. (2016), who proved that the problem is $N P$-hard. ${ }^{8}$

Batch learning. In this problem, the learning algorithm must use a batch of samples to compute one reserve price per bidder. This problem was studied by Morgenstern and Roughgarden (2015): Their "1-level auctions" correspond exactly to second-price auctions with non-anonymous reserve prices. ${ }^{9}$ Their work focuses on sample complexity bounds. Their learning algorithm uses an algorithm for offline optimization as a subroutine, but they do not address its computational efficiency.

Online vanishing-regret learning. The online learning algorithm must now compute one reserve price per bidder in each time step, and perform almost as well as the best fixed nonanonymous reserve prices in hindsight. This problem has not been previously considered.

We study the problem of computing and learning multiple reserve prices primarily because of its basic nature, but we note that non-anonymous reserve prices are used in practice (often in a disguised form, for better optics). For example, in sponsored search auctions a "quality score" is associated with each advertiser, and different quality scores translate to different effective reserve prices for different advertisers (see, e.g., Athey and Nekipelov (2010)). Along the same lines, in the recent FCC Incentive Auction (for re-allocating spectrum licenses from TV broadcasters to mobile broadband companies), a particular formula is used to set different opening bids for different participants, depending on the market share and geographic location of the broadcaster (see, e.g., Cramton et al. (2015)).

\footnotetext{
${ }^{5}$ These are called "eager reserves" by Dhangwatnotai et al. (2015). With "lazy reserves," the winner is the highest bidder, if she clears her reserve (and no one, otherwise), and the selling price is the maximum of her reserve and the second-highest bid overall. Note that whenever a sale occurs with lazy reserves, a sale would also occur with eager reserves (but not vice versa). Eager reserves are superior from both a welfare and revenue standpoint; see Leme et al. (2016) for these and many other interesting comparisons.

${ }^{6}$ These results are extended to lazy monopoly reserves by Dhangwatnotai et al. (2015).

${ }^{7}$ At a high level, the problem of setting prices to maximize revenue with respect to known valuations is reminiscent of the algorithmic pricing literature, beginning with Guruswami et al. (2005).

${ }^{8}$ The analogous problem with lazy reserve prices is easy (Leme et al. 2016).

${ }^{9}$ The problem of batch learning a near-optimal auction that need not be reserve-price-based is studied implicitly by Elkind (2007) and explicitly by Cole and Roughgarden (2014), Devanur et al. (2016), and Gonczarowski and Nisan (2017). Because there is no restriction on the produced auction, these learning algorithms are computationally efficient.
} 
More generally, our problems fall under the umbrella of computing or learning "best-in-class" mechanisms from a restricted class. There are not many opportunities in practice to design an auction from scratch; more typically, there are a limited number of parameters of an alreadychosen auction format that can be tuned. For example, you can pick your opening bid in an eBay auction, but you're stuck with a Vickrey auction with an anonymous reserve price. "Best-in-class" mechanism design models these types of applications. ${ }^{10}$

\subsection{Our Results}

We first consider the offline optimization problem MAXimizing Multiple Reserves (MMR) in general matroid environments (see Section 2 for background). The input is $m$ valuation profiles $\mathbf{v}^{1}, \ldots, \mathbf{v}^{m}$, indexed by the same $n$ bidders. The goal is to compute the vector $\mathbf{r}$ of reserve prices that maximizes the total revenue obtained on these profiles by the Vickrey-Clarke-Groves mechanism with reserves $\mathbf{r}$. We prove that the problem is $A P X$-hard (i.e., hard to approximate better than some fixed constant), even in the special case of single-item environments, and give a polynomial-time $\frac{1}{2}$-approximation algorithm for it in arbitrary matroid environments. ${ }^{11}$

This immediately implies a polynomial-time approximate learning algorithm for the batch learning problem, which (with high probability) computes reserve prices leading to expected revenue (w.r.t. the unknown distribution $F$ ) at least $\frac{1}{2}-\epsilon$ times that obtained with the best reserve prices for $F$.

We then consider the online vanishing-regret learning problem. The $\frac{1}{2}$-approximation algorithm for the offline problem does not automatically imply an approximate vanishing-regret online learning algorithm. ${ }^{12}$ We show how to exploit the special structure of the MMR problem to translate our offline approximation algorithm into an online learning algorithm that achieves time-averaged revenue at least $\frac{1}{2}$ times that of the best fixed reserve prices in hindsight, less an $o(1)$ error term (as $T \rightarrow \infty)$. This positive result applies to arbitrary matroid environments. ${ }^{13}$ On the negative side, we show that, quite generally, computational hardness for the offline optimization problem translates to computational hardness for obtaining vanishing time-averaged regret. ${ }^{14}$ (This is not obvious, because an online algorithm might achieve high revenue with different reserves at different times, while never figuring out the best fixed reserves in hindsight.) This general translation may be of independent interest. In any case, our hardness result for the MMR problem carries over to the problem of online vanishing-regret learning, even in the special case of single-item auction environments.

\footnotetext{
${ }^{10}$ Without any restriction on the mechanism, the optimal solution to the offline optimization problem can be computed in polynomial time using linear programming (Dobzinski et al. 2011). One rarely has the luxury of implementing such an opaque and distribution-specific mechanism.

${ }^{11}$ Both our approximation algorithm and analysis approach bear some resemblance to the lookahead auction of Ronen (2001) and its extension to matroids by Chawla et al. (2014). (The uniform distribution over the valuation profiles in an offline instance can be thought of as a correlated valuation distribution.) We require a different argument, because we restrict our solution to a fixed vector of reserve prices; the lookahead auction uses "conditional reserve prices." To see the difference, suppose there are two bidders and two valuation profiles, $(1,2)$ and $(2,4)$. The lookahead auction extracts the full welfare with respect to the uniform distribution over the two profiles (by always charging the second bidder twice the first bid), and no fixed set of reserve prices can compete.

${ }^{12}$ For linear optimization problems, there is such a black-box reduction (Kakade et al. 2009). But in the MMR problem, the revenue is not a linear function of the reserves or of the valuations.

${ }^{13}$ After the conference version of this article, Roughgarden and Wang (2016) and Dudik et al. (2017) studied such "onlineto-offline reductions" in more general settings. One of their results gives conditions on an approximation algorithm under which it can be translated into an equally good online learning algorithm, and it includes our Theorem 4.1 as a special case. ${ }^{14}$ Related results, based on somewhat different arguments, were developed independently by Daskalakis and Syrgkanis (2016), in the context of utility-maximization for a bidder (rather than revenue-maximization for a seller) in simultaneous second-price auctions.
} 


\section{PRELIMINARIES}

\subsection{Matroid Environments}

For our purposes, an environment is defined by a set $E$ of bidders and a non-empty collection $I \subseteq 2^{E}$ of feasible sets of bidders, which are the subsets of bidders that can simultaneously "win." For example, in a $k$-unit auction with unit-demand bidders, $\mathcal{I}$ is all subsets of $E$ that have size at most $k$. Each bidder has a private valuation for winning. Depending on the setting, bidders' valuations can be drawn from a (possibly unknown) distribution, or arbitrary.

We consider matroid environments, where (i) the set system $(E, \mathcal{I})$ is downward-closed, meaning that if $T \in \mathcal{I}$ and $S \subseteq T$, then $S \in \mathcal{I}$; and (ii) the exchange property holds, stating that whenever $S, T \in \mathcal{I}$ with $|T|<|S|$, there is some $i \in S \backslash T$ such that $T \cup\{i\} \in \mathcal{I}$. Examples of matroid environments include digital goods (where $\mathcal{I}=2^{E}$ ), $k$-unit auctions (where $\mathcal{I}$ is all subsets of size at most $k$ ), and certain unit-demand matching markets (corresponding to transversal matroids).

\subsection{The VCG Mechanism with Non-Anonymous Reserves}

Name the bidders $E=\{1,2, \ldots, n\}$. A (deterministic) mechanism $\mathcal{M}$ comprises an allocation rule $\mathbf{x}$ that maps every bid vector $\mathbf{b}$ to a characteristic vector of a feasible set (in $\{0,1\}^{n}$ ), and a payment rule $\mathbf{p}$ that maps every bid vector $\mathbf{b}$ to a non-negative payment vector in $[0, \infty)^{n}$. We assume that every bidder $i$ aims to maximize its quasi-linear utility $u_{i}(\mathbf{b})=v_{i} \cdot x_{i}(\mathbf{b})-p_{i}(\mathbf{b})$, where $v_{i}$ is its private valuation for winning. We call a mechanism $\mathcal{M}$ truthful if for every bidder $i$ and fixed bids $\mathbf{b}_{-i}$ of the other bidders, bidder $i$ maximizes its utility by setting its bid $b_{i}$ to its private valuation $v_{i}$. Since we only consider truthful mechanisms, in the rest of the article, we use valuations and bids interchangeably. The efficiency or welfare of the outcome of a mechanism is the sum of the winners' valuations, and the revenue is the sum of the winners' payments.

The VCG mechanism chooses the feasible set $S \in \mathcal{I}$ that maximizes the welfare $\sum_{i \in S} v_{i}$. Each winner in the mechanism pays the smallest bid at which she would continue to win; this results in a truthful mechanisms. In a single-item setting, this is just the second-price auction.

Let $r_{i}$ be a reserve price for bidder $i$. The VCG mechanism with reserves $\mathbf{r}$ works as follows, given bids v: (1) remove all bidders $i$ with $v_{i}<r_{i}$; (2) run the VCG mechanism on the remaining bidders to determine the winners; (3) charge each winning bidder $i$ the larger of $r_{i}$ and its VCG payment in step (2). This is again a truthful mechanism (for any $\mathbf{r}$ ).

Matroid environments are special in that the VCG mechanism can be implemented using a greedy algorithm. Specifically, to choose the winners: sort the bidders in order of decreasing bids, and in one pass in this order, add a bidder to the winner set if and only if doing so preserves feasibility. It is well known and easy to prove that this algorithm computes the welfare-maximizing outcome.

This greedy algorithm also reveals what the payments are. Consider some winning bidder $i$. As a thought experiment, imagine re-running the greedy algorithm without $i$, and let $f(i)$ be the first winning bidder after which it would be infeasible to add $w$ the winner set (if any). The VCG payment of $i$ is the valuation of $f(i)$, or zero if $f(i)$ does not exist.

\section{THE OFFLINE OPTIMIZATION PROBLEM: ALGORITHMS}

This section studies offline optimization (the second problem of the four described in the Introduction). The offline MMR problem is interesting in its own right, and algorithms for it also serve as a useful starting point for designing online MMR algorithms. We warm up with the single-item case (Section 3.1) and then address the general matroid environment case (Section 3.2). 


\subsection{Warm-Up: The Single-Item Case}

In the single-item special case of the MMR problem, the input is valuation profiles $\mathbf{v}^{1}, \ldots, \mathbf{v}^{m}$, and the goal is to choose (non-anonymous) reserve prices $\mathbf{r}$ to maximize the average revenue of a second-price auction with reserves $\mathbf{r}$ on these profiles. Without loss of generality, each reserve price $r_{i}$ is equal to the valuation of bidder $i$ in one of the $m$ valuation profiles. (This still leaves a search space of $m^{n}$ reserve price vectors.)

Observe the tension in setting the reserve price $r_{i}$ for a bidder $i$ : Increasing it may increase revenue on profiles where $i$ is a winner (provided $r_{i}$ stays below $v_{i}$ ), but it may also decrease revenue if $r_{i}$ passes $v_{i}$, even when $i$ is only the second-highest bidder $\left(v_{i}\right.$ was previously setting the price of the winner). The following algorithm balances these considerations:

\section{MMR Algorithm (Single-Item Case)}

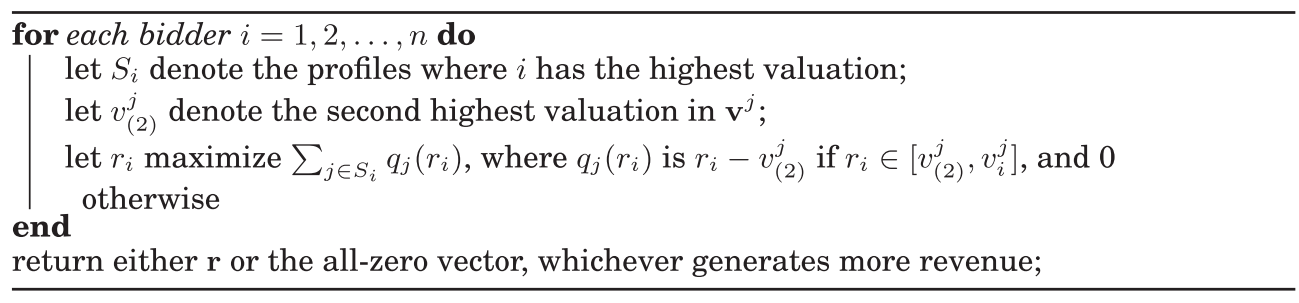

In words, $q_{j}\left(r_{i}\right)$ represents the additional revenue obtained by the reserve price $r_{i}$ from bidder $i$ on day $j$, above and beyond the revenue already obtained by a reserve price of 0 .

This algorithm can be implemented in polynomial time. Each reserve price $r_{i}$ can be computed independently, and there are only $m$ different relevant choices for each.

LEMMA 3.1. The algorithm above is a $\frac{1}{2}$-approximation algorithm for the MMR problem in singleitem environments.

Proof. Fix an input $\mathbf{v}^{1}, \ldots, \mathbf{v}^{m}$ and let $\mathbf{r}^{*}$ denote the optimal reserve prices. The revenue obtained from a valuation profile $\mathbf{v}^{j}$ with $j \in S_{i}$ is at most $\max \left\{v_{(2)}^{j}, r_{i}^{*}\right\}$. (If the item is sold to someone other than the highest bidder, then the selling price is at most $v_{(2)}^{j}$.) Overall, the optimal revenue can be upper bounded by

$$
\sum_{i=1}^{n} \sum_{j \in S_{i}} \max \left\{v_{(2)}^{j}, r_{i}^{*}\right\} \leq \sum_{j=1}^{m} v_{(2)}^{j}+\sum_{i=1}^{n} \sum_{j \in S_{i}} q_{j}\left(r_{i}^{*}\right) .
$$

The all-zero reserves obtain revenue equal to the first term on the right-hand side. The reserves $\mathbf{r}$ computed by the algorithm above obtain, by construction, revenue that is at least the second term on the right-hand side (with $r_{i}$ chosen to maximize the $i$ th inner sum). The better of these two reserve price vectors earns revenue at least $\frac{1}{2}$ times the maximum possible.

Example 3.2. Our analysis in Lemma 3.1 is tight: For every $\epsilon>0$, there is an input where the algorithm achieves less than $(1 / 2+\epsilon)$ times the optimal revenue. The bad input is as follows: let $n$ be an integer to be chosen later, and let there be two bidders and $n$ valuation profiles. The first bidder has valuation $n$ on the first day, valuation 0 on the second day, and valuation $1+1 / n$ on every other day. The second bidder has valuation 0 on the first day, valuation $1+1 / n$ on the second day, and valuation 1 on every other day. For this input, the all-zero vector will achieve 
a revenue of $(n-2)$. Also, $\mathbf{r}$ will be the vector $(n, 1+1 / n)$, which achieves a revenue of $(n+1+$ $1 / n)$. However, choosing $\mathbf{r}^{\star}$ to be $(n, 1)$ results in a revenue of $(2 n-1)$. When $n>\frac{5}{4} \epsilon^{-1}+\frac{1}{2}$, the algorithm's revenue is less than $(1 / 2+\epsilon)$ times the optimal revenue.

\subsection{An Offline MMR Algorithm}

We now consider matroid environments. The new complication is that there are multiple winners, and we need to determine the relationship between the winners in the VCG mechanism (without reserves) and the winners in an optimal solution.

The generalization of our previous algorithm is as follows:

\section{MMR Algorithm (Matroid Case)}

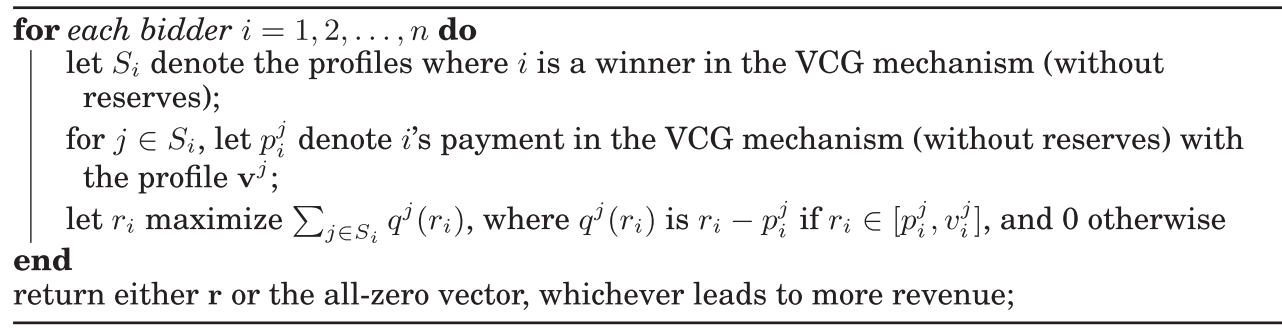

As in the single-item special case, it is straightforward to implement this algorithm so it runs in polynomial time.

As a lead-up to the analysis, the next three propositions review well-known properties of matroids and the VCG mechanism (see, e.g., Talwar (2003)).

Proposition 3.3 (Schrijver (2003), Corollary 39.12A). Let $W, W^{\prime}$ be feasible sets of size $k$ in a matroid $M$. Then there is a bijection $f: W^{\prime} \backslash W \rightarrow W \backslash W^{\prime}$ such that for every $i \in W^{\prime} \backslash W$, the set $W \backslash\{f(i)\} \cup\{i\}$ is a feasible set in $M$.

From the definition of VCG payments, we have:

Proposition 3.4. Suppose that the winners in the VCG mechanism with zero reserves are $W$, and that $i \in W$ but $j \notin W$, and that $W \backslash\{i\} \cup\{j\}$ is a feasible set. Then the VCG payment of $i$ is at least the valuation of $j$.

The following proposition explains what happens when a winner is removed from the auction:

Proposition 3.5. Suppose the current winners of a matroid auction are $W$, and we then remove some winner $w \in W$ from the auction. Then the new set of winners will be either be of the form $W \backslash\{w\} \cup w^{\prime}$ where $w^{\prime}$ is a new winner in $E \backslash W$, or of the form $W \backslash\{w\}$.

Lemma 3.6 (Revenue Decomposition Lemma). Consider a valuation profile $\mathbf{v}$ and reserve prices $\mathbf{r}^{\star}$. Let the winners of the VCG mechanism with profile $\mathbf{v}$ and no reserves be $W$, with $p_{i}$ denoting the payment of $i$. Then, the revenue of $\mathbf{r}^{\star}$ on $\mathbf{v}$ is at most

$$
\sum_{i \in W} p_{i}+\sum_{i \in W} q\left(r_{i}^{*}\right)
$$

where $q\left(r_{i}^{*}\right)$ is $r_{i}^{*}-p_{i}$ if $r_{i} \in\left[p_{i}, v_{i}\right]$, and 0 otherwise.

Proof. The winning set $W$ with zero reserves is a basis of the matroid of feasible subsets. The reserves $\mathbf{r}^{\star}$ result in some set of winners $W^{\star}$. Extend $W^{\star}$ to a basis using the exchange property 
(Section 2), and apply Proposition 3.3 to get a bijection from a superset of $W^{\star}$ to $W$. Ignoring elements not in $W^{\star}$, we get a injection $f$ from $W^{\star}$ to $W$. Furthermore, we are guaranteed that for every bidder $i \in W^{\star}, W \backslash\{f(i)\} \cup\{i\}$ is feasible. Applying Proposition 3.4, this means that whenever $f(i) \neq i, p_{f(i)}$ is at least the valuation of $i$.

Next, consider a bidder $i \in W$. We claim that increasing the reserves for bidders other than $i$ (from 0 to some positive amount) can never cause $i$ to lose or to pay more than $p_{i}$. For the first statement, the only effect of reserve prices on the winning set is to remove bidders before invoking the greedy algorithm. By Proposition 3.5, removing a winner and re-running the greedy algorithm simply causes them to be replaced (i.e., no other bidders are removed as a consequence). Trivially, removing a nonwinner has no effect on the winning set. Thus, if $i$ 's reserve is not changed, it remains a winner. Similarly, removing other bidders can only decrease the valuation of the losing bidder that sets $i$ 's price (recall Section 2).

These two facts imply that, for every winner $i \in W^{\star}$, we can use $f$ to find a winner $f(i) \in W$ such that either (i) $i$ 's valuation, and hence contribution to the revenue of $\mathbf{r}^{\star}$, is at most $p_{f(i)}$ or (ii) $f(i)=i$ and $i$ only pays more than $p_{i}$ to the extent that $r_{i}^{\star}$ is larger than $p_{i}$ (while also being at most $v_{i}$ ). The quantity in (ii) is precisely $q\left(r_{i}^{*}\right)$. Because $f$ is an injection, we can sum over all $w \in W^{\star}$ to get the inequality in the lemma statement.

The lemma easily implies that our algorithm is a $\frac{1}{2}$-approximation.

THEOREM 3.7. The algorithm above is a $\frac{1}{2}$-approximation algorithm for the MMR problem in matroid environments.

Proof. Fix an input $\mathbf{v}^{1}, \ldots, \mathbf{v}^{m}$ and let $\mathbf{r}^{*}$ denote the optimal reserve prices. Let $W_{j}$ denote the winners in the VCG mechanism with profile $\mathbf{v}^{j}$ and no reserves, and $p_{i}^{j}$ the payment made by a bidder $i \in W_{j}$. Applying Lemma 3.6 to each profile $\mathbf{v}^{j}$ and summing over $j$, the total revenue obtained by $\mathbf{r}^{*}$ is at most

$$
\sum_{j=1}^{m}\left(\sum_{i \in W_{j}} p_{i}^{j}+\sum_{i \in W_{j}} q^{j}\left(r_{i}^{*}\right)\right)=\sum_{j=1}^{m} \sum_{i \in W_{j}} p_{i}^{j}+\sum_{i=1}^{n} \sum_{j \in S_{i}} q^{j}\left(r_{i}^{*}\right),
$$

where $S_{i}$ and $q^{j}$ are defined as in the algorithm description. The all-zero reserves obtain total revenue equal to the first term on the right-hand side. The reserves $\mathbf{r}$ computed by the algorithm above earn revenue that is at least the second term on the right-hand side (again, with $r_{i}$ chosen to maximize the $i$ th inner sum). The better of these two reserve price vectors earns revenue at least $\frac{1}{2}$ times the maximum possible.

\subsection{Consequences for Batch Learning}

Recall the batch learning problem mentioned in the Introduction: Valuation profiles $\mathbf{v}^{1}, \ldots, \mathbf{v}^{m}$ are sampled i.i.d. from an unknown distribution $F$ and given as a batch to a learning algorithm. The responsibility of the learning algorithm is to output non-anonymous reserve prices $\mathbf{r}$ that earn expected revenue (on a new i.i.d. draw from $F$ ) within $\epsilon$ of that obtained by the optimal nonanonymous reserve prices (for $F$ ). Morgenstern and Roughgarden (2015) show that, informationtheoretically, $m=\Omega\left(\frac{H^{2}}{\epsilon^{2}} n \log n\right)$ samples are enough to in principle compute such reserve prices in matroid environments (with high probability over the samples, and where $H$ is a bound on the maximum valuation). This guarantee is realized by the "empirical risk minimizing (ERM)" algorithm, which simply solves the offline optimization problem (using the samples as input) and returns the result. Morgenstern and Roughgarden (2015) did not address computational complexity issues in their work. Since the offline optimization problem is NP-hard to approximate to within 
some constant (Theorem 5.2 below), the ERM algorithm cannot be implemented in polynomial time (unless $P=N P$ ).

On the positive side, the same machinery (based on "uniform convergence") used to establish the guarantee of the ERM algorithm applies to approximation algorithms for the offline optimization problem. Thus, our $\frac{1}{2}$-approximation algorithm for the MMR problem (Theorem 3.7) can be used as a black box to efficiently compute, from $m=\Omega\left(\frac{H^{2}}{\epsilon^{2}} n \log n\right)$ i.i.d. samples from an unknown distribution $F$, reserve prices that obtain expected revenue at least half that of the maximum possible (with high probability, and less $\epsilon$ ).

\section{ONLINE LEARNING ALGORITHMS}

This section considers the (adversarial) online vanishing-regret learning problem, the fourth of the problems described in the Introduction.

\subsection{Regret-minimization}

Here, valuation profiles $\mathbf{v}^{1}, \ldots, \mathbf{v}^{T}$ arrive one at a time (indexed by the same set of $n$ bidders in a matroid environment), and at time $t$ a learning algorithm chooses reserve prices $\mathbf{r}^{t}$ as a function of the previously seen profiles $\mathbf{v}^{1}, \ldots, \mathbf{v}^{t-1}$. We use $R\left(\mathbf{r}^{t}, \mathbf{v}^{t}\right)$ to denote the revenue earned by the VCG mechanism with reserve prices $\mathbf{r}^{t}$ on the valuation profile $\mathbf{v}^{t}$. The goal is to choose reserve prices over time so the time-averaged revenue is almost as high as that achieved by the best fixed reserve prices in hindsight. Formally, the regret of a sequence $\mathbf{r}^{1}, \ldots, \mathbf{r}^{T}$ of reserve prices with respect to a sequence of valuation profiles $\mathbf{v}^{1}, \ldots, \mathbf{v}^{T}$ is

$$
\max _{\mathbf{r}} \frac{1}{T} \sum_{t=1}^{T} R\left(\mathbf{r}, \mathbf{v}^{t}\right)-\frac{1}{T} \sum_{t=1}^{T} R\left(\mathbf{r}^{t}, \mathbf{v}^{t}\right),
$$

and the goal is drive this quantity toward 0 as quickly as possible (as $T$ grows large). Since regret corresponds to additive error, we normalize valuations to lie in the range $[0,1]$.

We prove in Section 6 that this notion of regret is too optimistic. Our main positive result is an online learning algorithm with vanishing $\alpha$-regret, a quantity defined by Kakade et al. (2009) to introduce approximation into vanishing-regret guarantees:

$$
\alpha \cdot \max _{\mathbf{r}} \frac{1}{T} \sum_{t=1}^{T} R\left(\mathbf{r}, \mathbf{v}^{t}\right)-\frac{1}{T} \sum_{t=1}^{T} R\left(\mathbf{r}^{t}, \mathbf{v}^{t}\right) .
$$

\subsection{An Online MMR Algorithm}

We now work toward an algorithm for the online setting with good $\alpha$-regret. A natural idea is to apply the existing machinery for translating offline $\alpha$-approximation algorithms into online learning algorithms with vanishing $\alpha$-regret. However, these techniques do not immediately apply in our setting. First, the number of actions (corresponding to reserve price vectors) is exponential in $n$, so we cannot separately track the past performance of each reserve price vector. The wellknown "follow-the-perturbed-leader (FTPL)" algorithm of Kalai and Vempala (2003) (based on the idea of Hannan (1957)) can be used to translate certain $\alpha$-approximation algorithms to online no$\alpha$-regret algorithms for arbitrary action spaces (subsets of $\mathbb{R}^{n}$ ), provided the payoff in each time step is a linear function of the chosen action. In our setting, the corresponding function $R\left(\mathbf{r}^{t}, \mathbf{v}^{t}\right)$ is not linear in $\mathbf{r}^{t}$ or $\mathbf{v}^{t}$. Nonetheless, we show how to combine the main ideas of the FTPL algorithm 
and of our offline approximation algorithm in Section 3 to obtain an online learning algorithm with vanishing $\frac{1}{2}$-regret. ${ }^{15}$

Our learning algorithm, defined for an arbitrary matroid environment, is given on the next page. It is straightforward to implement this algorithm so it runs in $O(n \sqrt{T})$ at each time step, and $O\left(n T^{3 / 2}\right)$ time overall. A key point is that each reserve price $r_{i}^{t}$ is determined independently for each bidder $i$, enabling us to keep track of only $K n$ quantities (as opposed to a quantity for each of the $K^{n}$ reserve price vectors). ${ }^{16}$

The analysis challenge is to show that our online algorithm earns revenue comparable to each term of the Revenue Decomposition Lemma (Lemma 3.6), despite having to produce reserve prices before seeing the valuation profile for which they will be used.

\section{MMR Algorithm (Online)}

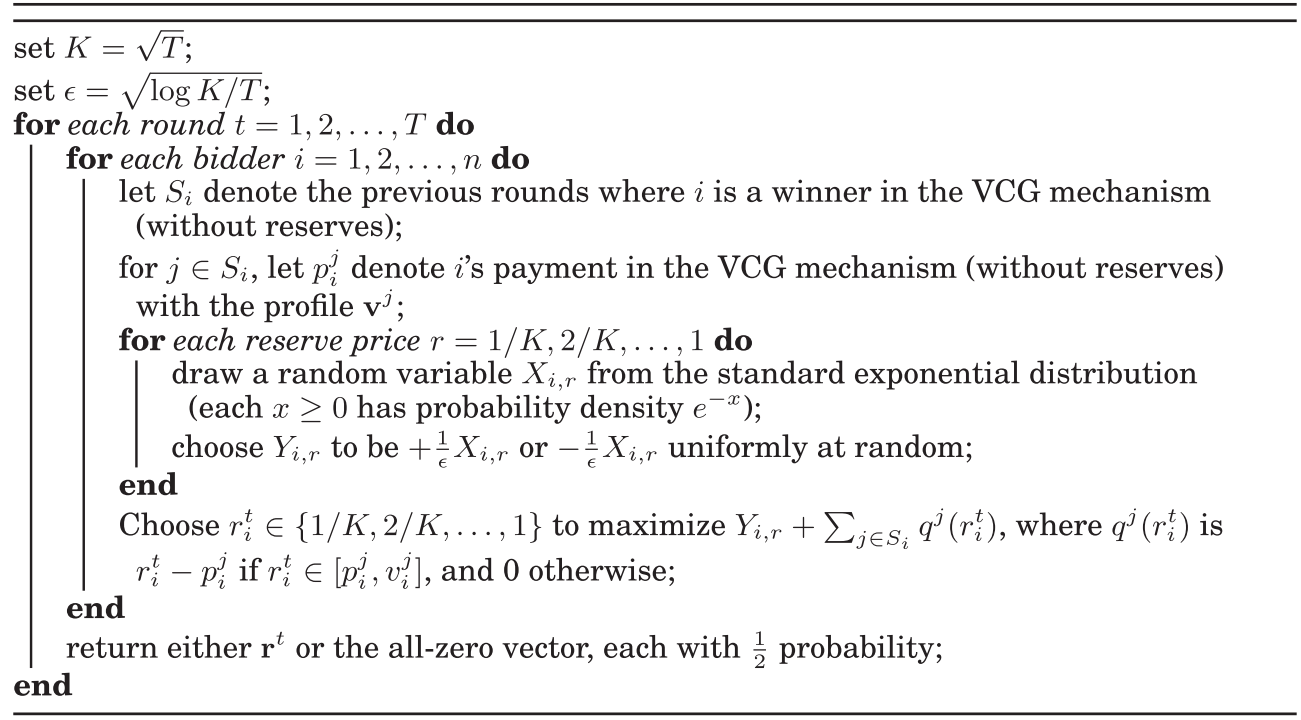

TheOREM 4.1. The $\frac{1}{2}$-regret of the online learning algorithm above is $O(n \sqrt{\log T / T})$.

Proof. Fix an input sequence $\mathbf{v}^{1}, \ldots, \mathbf{v}^{T}$ and let $\mathbf{r}^{\star}$ denote the optimal reserve prices. Let $W_{t}$ denote the winners in the VCG mechanism with profile $\mathbf{v}^{t}$ and no reserves, and $p_{i}^{t}$ the payment made by a bidder $i \in W_{t}$. Lemma 3.6 again implies, after summing over $t$, that the total revenue obtained by $\mathbf{r}^{\star}$ is at most

$$
\sum_{t=1}^{T}\left(\sum_{i \in W_{t}} p_{i}^{t}+\sum_{i \in W_{t}} q^{t}\left(r_{i}^{*}\right)\right)=\sum_{t=1}^{T} \sum_{i \in W_{t}} p_{i}^{t}+\sum_{i=1}^{n} \sum_{t \in S_{i}} q^{t}\left(r_{i}^{*}\right),
$$

where $S_{i}$ and $q^{t}$ are defined as in the algorithm description. Choosing the all-zero reserve prices every round obtains total revenue equal to the first term on the right-hand side. We will prove that

\footnotetext{
${ }^{15}$ This use of the FTPL algorithm in online auction design is reminiscent of Blum and Hartline (2005); the problem studied there corresponds to a single-bidder setting in the present setup.

${ }^{16}$ The algorithm is described for the case where the time horizon $T$ is known in advance. Standard doubling arguments extend the algorithm and analysis to the case of an a priori unknown time horizon (see, e.g., Cesa-Bianchi and Lugosi (2006)).
} 
choosing the $\mathbf{r}^{t}$ computed by the algorithm every round obtains total expected revenue equal to the second term on the right-hand side, less an error of $O(n \sqrt{T \log T})$.

The algorithm limits itself to reserve prices from the set $\{1 / K, 2 / K, \ldots, 1\}$ rather than $[0,1]$. Then, for each bidder $i$, it tries to find a reserve $r_{i}$ that maximizes $\sum_{t \in S_{i}} q^{t}\left(r_{i}\right)$, with perturbations as in the FTPL algorithm.

We use a result due to Kalai and Vempala (2003). They considered an online decision-making setting with $K$ actions (a.k.a. "experts") and an adversary that picks a cost vector (mapping each action to $[0,1])$ in every round. They analyzed the FTPL algorithm, which at each time step computes the cumulative cost of every action to-date, adds noise to each total (distributed like the $Y_{i, r}$ 's in the algorithm above), and plays the action with the smallest perturbed cost. They prove that the expected cost of this FTPL algorithm is at most the cost of the best fixed expert plus

$$
\epsilon T+\frac{O(\log K)}{\epsilon} .
$$

Our algorithm treats each possible reserve as an action and considers $1-q^{t}$ to be the cost of choosing an expert in round $t$. Note that our algorithm runs the FTPL algorithm every round for bidder $i$, but $S_{i}$ only increases when $i$ turned out to be a winner in the VCG mechanism without reserves. When we consider only rounds where $i$ is a winner in the VCG mechanism without reserves, the Kalai-Vempala guarantee translates to:

$$
\mathbb{E}\left[\sum_{t \in S_{i}} q^{t}\left(r_{i}^{t}\right)\right] \geq \sum_{t \in S_{i}} q^{t}\left(r_{i}^{\star}\right)-\epsilon T-\frac{O(\log K)}{\epsilon} \geq \sum_{t \in S_{i}} q^{t}\left(r_{i}^{\star}\right)-O(\sqrt{T \log T}),
$$

with second inequality following from our choices of $\epsilon$ and $K$. However, the $r_{i}^{\star}$ in this equation is limited to the set $\{1 / K, 2 / K, \ldots, 1\}$. This restriction costs at most $\frac{T}{K}=\sqrt{T}$ revenue (by rounding $r_{i}^{*}$ down to the nearest multiple of $\left.1 / K\right)$, which folds into the $O(\sqrt{T \log T})$ term.

Summing over all bidders $i$, choosing $\mathrm{r}^{t}$ every round gives expected revenue at least

$$
\sum_{i=1}^{n} \sum_{t \in S_{i}} q^{t}\left(r_{i}^{\star}\right)-O(n \sqrt{T \log T})
$$

We conclude that randomly returning either $\mathbf{r}^{t}$ or the all-zero vector at each round $t$ yields expected revenue at least $\frac{1}{2}$ times the maximum revenue achieved by any fixed set of reserve prices, less an error of $O(n \sqrt{T \log T})$. Hence, our algorithm has $O(n \sqrt{\log T / T})$ (time-averaged) $\frac{1}{2}$-regret, as claimed.

\section{OFFLINE LOWER BOUND}

In this section, we prove that the MMR problem is $N P$-hard to approximate within a $(1+\epsilon)$ factor for some constant $\epsilon>0$, even in the special case of single-item environments. Section 6 extends this hardness result to the online vanishing-regret learning problem.

Our proof approach is to provide an $L$-reduction, in the sense of Papadimitriou and Yannakakis (1988), from DominatingSeT on graphs of bounded degree to the MMR problem.

Definition 5.1 (Papadimitriou and Yannakakis (1988)). Suppose $\Pi$ and $\Pi^{\prime}$ are two maximization problems. We say $\Pi$ L-reduces to $\Pi^{\prime}$ if there are poly-time algorithms $f, g$ and constants $\alpha, \beta>0$ such that given an instance $I$ of $\Pi$ :

(1) Algorithm $f$ produces an instance $I^{\prime}$ of $\Pi^{\prime}$ such that $O P T\left(I^{\prime}\right) \leq \alpha O P T(I)$.

(2) Given a solution of $I^{\prime}$ with cost $c^{\prime}$, algorithm $g$ produces a solution of $I$ with $\operatorname{cost} c$ with $|c-O P T(I)| \leq \beta\left|c^{\prime}-O P T\left(I^{\prime}\right)\right|$. 
$2 n$ valuation profiles

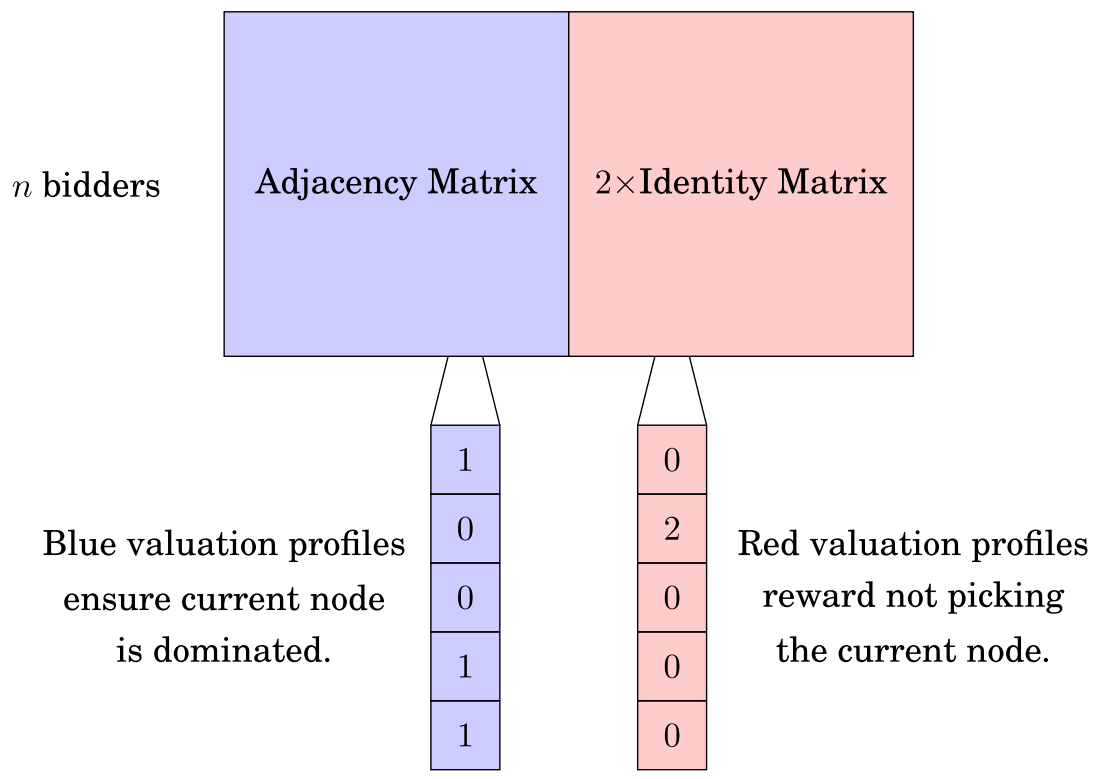

Fig. 1. Transforming a dominating set instance into an MMR instance.

This definition is designed so $L$-reductions compose and if $\Pi L$-reduces to $\Pi^{\prime}$, then a $(1+\epsilon)$ approximation for $\Pi^{\prime}$ yields a $(1+\alpha \beta \epsilon)$-approximation for $\Pi$. The DominatingSET problem on graphs of bounded degree is hard to approximate to within some constant, so our $L$-reduction proves the same hardness result for the MMR problem.

Theorem 5.2. DominatingSet on graphs of bounded degree B L-reduces to the MMR problem in single-item environments with $\alpha=3 B+2$ and $\beta=1$.

Proof. Suppose we have a Dominating Set- $B$ instance $G=(V, E)$. Suppose it has $n$ nodes and the smallest dominating set uses $k$ nodes. We produce an MMR instance with $n$ bidders and $2 n$ valuation profiles, which is illustrated in Figure 1. The profiles are divided into two groups: $n$ blue profiles and $n$ red profiles. For convenience, we allow valuations in $[0,2]$ rather than $[0,1]$ (by scaling, it doesn't matter). The valuation of bidder $i$ in the $j$ th blue profile will be 1 if $i=j$ or $(i, j) \in E$ and 0 otherwise. The valuation of bidder $i$ in the $j$ th red profile will be 2 if $i=j$ and 0 otherwise.

For every bidder, the only valuations they ever have are 0,1 , and 2 . Hence, the only possible reserve choices for each bidder are 1 or 2 (we can always round up to the nearest valuation). We associate a reserve price of 1 with choosing the node to be in the dominating set and a reserve price of 2 with not choosing the node. In a blue profile, a bidder can only clear a reserve of 1 . In a red profile, the relevant bidder faces no competition, and hence a second-price auction with research only extracts the revenue from them by choosing the reserve of 2 . The blue profiles encode the dominating set constraint, while the red profiles encode the objective to minimize nodes chosen.

Suppose there is a dominating set of size $k$. Choosing the associated reserve prices, as described above, achieves a revenue of $3 n-k$ : We achieve a revenue of 1 in the blue profiles, since we cover them all by definition, a revenue of 1 from each red profile corresponding to one of the $k$ chosen nodes, and a revenue of 2 from each of the other $n-k$ red profiles. 
Since every node in the original problem covered at most $B+1$ nodes, the original optimum was at least $n /(B+1)$. Combining this bound with our argument above, the optimal revenue is at most $\frac{3 B+2}{B+1} n$. Hence, we can choose $\alpha=3 B+2$.

Next, we show that if there are non-anonymous reserve prices that get within $c$ of the new optimum, then we can produce a dominating set that gets within $c$ of the old optimum (with a polynomial-time algorithm). Suppose we achieve a revenue of $3 n-k$ for some $k$. We claim that we can assume that we achieve a revenue of 1 in every blue profile. For if we do not, then consider any bidder who has a valuation of 1 in that profile. Changing their reserve to 1 nets an additional 1 revenue in this profile, and loses at most 1 revenue (from their red profile). Hence, in polynomial time, we can transform a solution to one that only has more revenue and achieves a revenue of 1 every blue profile. But then our association of nodes with reserve prices gives us a dominating set of size at most $k$. This completes the proof with $\beta=1$.

Tight approximation bounds are not known for bounded-degree dominating set and related problems, and as a result there is a gap between our lower bound in Theorem 5.2 and our $\frac{1}{2}$ approximation algorithm. Using the state-of-the-art result of Chlebik and Chlebikova (2008) (with $B=5$ ), we get that the MMR problem cannot be approximated better than a factor of $\frac{884}{885}$. This inapproximability result holds for generally for estimating the value of an optimal solution to the MMR problem; this will be useful in the next section.

Corollary 5.3. It is NP-hard to distinguish between single-item MMR instances in which the maximum-possible revenue is at least a given parameter $X$, and those instances in which the maximum-possible revenue is at most $\frac{884}{885} X$, even when $X \geq \frac{m}{2}$.

\section{ONLINE LOWER BOUNDS}

\subsection{Online MMR Lower Bound}

Our hardness result for offline optimization (Theorem 5.2) translates to an analogous hardness result for online learning. This is not immediately obvious, because an online learning algorithm could conceivably achieve high revenue with different reserves at different times, while never figuring out the best fixed reserves in hindsight. Our randomized polynomial-time reduction is the following (where $\left.\alpha \in\left(\frac{884}{885}, 1\right]\right)$ :

\section{MMR Reduction (Offline to Online)}

Input: valuation profiles $\mathbf{v}^{1}, \ldots, \mathbf{v}^{m}$ and target $X$;

let $A$ be the online algorithm and $f(T)$ its $\alpha$-regret after $T$ rounds; choose the smallest $T$ such that $f(T)$ is at most $\frac{1}{4} \cdot\left(\alpha-\frac{884}{885}\right)$;

for rounds $t=1,2, \ldots, T$ do

ask $A$ for its next choice of reserves $\mathbf{r}^{t}$ and then give it a uniformly random valuation profile from $\left\{\mathbf{v}^{1}, \ldots, \mathbf{v}^{m}\right\}$;

end

return YES if at least one of the $\mathbf{r}^{t}$ results in strictly more than $\frac{884}{885} X$ total revenue on $\left\{\mathbf{v}^{1}, \ldots, \mathbf{v}^{m}\right\}$, and NO otherwise;

We note that whenever the resulting offline algorithm says "yes" it must be correct-it finds a reserve price vector witnessing the lower bound on the optimal objective function value. Thus, the algorithm has one-sided error (with false negatives only). 
For the purposes of the following theorem, we say that an online learning algorithm has no $\alpha$-regret if for every fixed constant $\epsilon>0$, the number of rounds needed to drive the $\alpha$-regret down to $\epsilon$ is bounded by a polynomial in the number of bidders $n$. For example, any regret bound of the form $\operatorname{poly}(n) / T^{\delta}$ (for any fixed $\delta>0$ ) satisfies this condition.

THEOREM 6.1. For all constants $\alpha>\frac{884}{885}$, there is no polynomial-time algorithm for the online MMR problem with no $\alpha$-regret, unless $N P \subseteq R P$.

Proof. We show that if the conjectured online algorithm exists, then the reduction above distinguishes between single-item MMR instances in which the maximum-possible revenue is at least a given parameter $X$; and those instances in which the maximum-possible revenue is at most $\frac{884}{885} X$, where $X$ is at least $\frac{m}{2}$ (with constant one-sided error). By Corollary 5.3, this would imply $N P \subseteq R P$.

For the analysis, our proof begins with algorithm $A$, which has $f(T) \alpha$-regret, and gradually transforms it to the same algorithm produced by our reduction. Recall that the $\alpha$-regret bound means that

$$
\alpha \frac{1}{T} \sum_{t=1}^{T} R\left(\mathbf{r}^{\star}, \mathbf{v}^{t}\right)-\mathbb{E}\left[\frac{1}{T} \sum_{t=1}^{T} R\left(\mathbf{r}^{t}, \mathbf{v}^{t}\right)\right] \leq f(T),
$$

for every sequence $\mathbf{v}^{1}, \ldots, \mathbf{v}^{T}$, where the expectation is over the coin flips of $A$.

Suppose we now change the setup as follows: For simplicity, assume that $T$ is a multiple of $m$. Define the $m$-periodic regret as the regret if only rounds $1, m+1,2 m+1$, and so on, contribute to the regret computation. (The best fixed auction in hindsight is still computed with respect to the valuation profiles at all rounds, not just this subset of rounds.)

Derive algorithm $A^{\prime}$ for the new setup from $A$ as follows: in rounds $1, m+1, \ldots, A^{\prime}$ simulates $A$ and also feeds it the new valuation profile. On other rounds $A^{\prime}$ behaves arbitrarily and does not feed anything into algorithm $A$. We know $A$ has $f(T / m) \alpha$-regret with respect to the sequence it is given. But the best fixed auction for this subsequence is only better than the best fixed auction for the entire sequence. Hence, $A^{\prime}$ has $f(T / m) m$-periodic $\alpha$-regret with respect to the original sequence. That is:

$$
\alpha \frac{m}{T} \sum_{t=1}^{T / m} R\left(\mathbf{r}^{\star}, \mathbf{v}^{m t-m+1}\right)-\mathbb{E}\left[\frac{m}{T} \sum_{t=1}^{T / m} R\left(\mathbf{r}^{m t-m+1}, \mathbf{v}^{m t-m+1}\right)\right] \leq f(T / m) .
$$

Next, we define an online algorithm $A^{\prime \prime}$ that uses the same reserves $\mathbf{r}^{t}$ for $m$ rounds in a row before changing, and bound its $\alpha$-regret. At a round $1, m+1, \ldots, A^{\prime \prime}$ simulates $A^{\prime}$. Whatever auction $A^{\prime}$ recommends, $A^{\prime \prime}$ plays this auction for the next $m$ rounds. During these rounds, $A^{\prime \prime}$ receives $m$ valuation profiles. After these rounds finish, $A^{\prime \prime}$ feeds these $m$ valuation profiles into $A^{\prime}$, one at a time in a random order (uniform over all $m$ ! possible orderings).

We now bound the $\alpha$-regret of $A^{\prime \prime}$. Fix a sequence of valuations $\mathbf{v}^{1}, \ldots, \mathbf{v}^{T}$ that an adversary chooses for $A^{\prime \prime}$, with the best fixed reserves $\mathbf{r}^{\star}$. Notice that $A^{\prime \prime}$ will give these same valuations to $A^{\prime}$, but possibly out of order. However, reordering does not affect the best fixed reserves. Furthermore, the $m$-periodic $\alpha$-regret guarantee of $A^{\prime}$ holds with respect to any input sequence, and in particular any reordering of our input sequence.

Consider a block of valuations, $\mathbf{v}^{(k-1) m+1}, \ldots, \mathbf{v}^{k m}$ that $A^{\prime \prime}$ must choose the same reserves for. We can express the expected average difference between the revenue of $A^{\prime \prime}$ and $\alpha$ times the revenue of $\mathbf{r}^{\star}$ on the entire block as follows:

$$
\alpha \frac{1}{m} \sum_{t=(k-1) m+1}^{k m} R\left(\mathbf{r}^{\star}, \mathbf{v}^{t}\right)-\mathbb{E}\left[\frac{1}{m} \sum_{t=(k-1) m+1}^{k m} R\left(\mathbf{r}^{(k-1) m+1}, \mathbf{v}^{t}\right)\right],
$$


where $\mathbf{r}^{(k-1) m+1}$ is the auction recommended by $A^{\prime}$ at the beginning of the block and the expectation is over any internal coin flips of the algorithm (this quantity is independent of the ordering of the profiles in the block). We can express the expected difference between the revenue of $A^{\prime}$ and $\alpha$ times the revenue of $\mathbf{r}^{\star}$ on the very first valuation of this block as follows:

$$
\alpha \sum_{t=(k-1) m+1}^{k m} \operatorname{Pr}\left[\mathbf{v}^{t} \text { first }\right] \cdot R\left(\mathbf{r}^{\star}, \mathbf{v}^{t}\right)-\mathbb{E}\left[\sum_{t=(k-1) m+1}^{k m} \operatorname{Pr}\left[\mathbf{v}^{t} \text { first }\right] R\left(\mathbf{r}^{(k-1) m+1}, \mathbf{v}^{t}\right)\right],
$$

where the probabilities are over the random ordering and the expectation over the coin flips of the algorithm. Because the probability that any particular $\mathbf{v}^{t}$ comes first in this block is $\frac{1}{m}$, these two expressions are equal. This allows us to relate the $m$-periodic $\alpha$-regret of $A^{\prime}$ with the $\alpha$-regret of $A^{\prime \prime}$. Summing the first expression over all blocks and averaging by the number of blocks yields the $\alpha$-regret of $A^{\prime \prime}$. Summing the second expression over all blocks and averaging by the number of blocks yields the $m$-periodic $\alpha$-regret of $A^{\prime}$, averaged over all orderings. Since ( $m$-periodic) regret is defined with respect to worst-case inputs, this means the $\alpha$-regret of $A^{\prime \prime}$ is $f(T / m)$.

Unraveling the definitions of $A^{\prime \prime}$ and $A^{\prime}, A^{\prime \prime}$ really just samples one valuation profile from every block of $m$ and runs $A$, which is the same as the algorithm produced by our reduction. The reduction algorithm above, in effect, uses $A^{\prime \prime}$ to approximate the offline problem, giving it $T$ blocks, where each block is a copy of the $m$ valuation profiles in its offline input, for a total of $T m$ rounds given to $A^{\prime \prime}$, which will simulate $A$ for $T$ rounds. By our assumption on the regret guarantee, $T$ will be polynomial in $n$. By our choice of $T$, from the guarantee for $A^{\prime \prime}$, we know:

$$
\alpha \frac{1}{m} \underbrace{\frac{1}{T} \sum_{t=1}^{m T} R\left(\mathbf{r}^{\star}, \mathbf{v}^{t}\right)}_{O P T}-\mathbb{E}\left[\frac{1}{m T} \sum_{t=1}^{m T} R\left(\mathbf{r}^{t}, \mathbf{v}^{t}\right)\right] \leq \frac{1}{4} \cdot\left(\alpha-\frac{884}{885}\right),
$$

where $\mathbf{r}^{t}$ is the action played by $A^{\prime \prime}$ at time $t$, OPT denotes the offline optimum's revenue, and the expectation is over the coin flips of $A^{\prime \prime}$, and hence

$$
\mathbb{E}\left[\frac{1}{T} \sum_{t=1}^{T} \sum_{j=1}^{m} R\left(\mathbf{r}^{t}, \mathbf{v}^{j}\right)\right] \geq \alpha O P T-\frac{m}{4} \cdot\left(\alpha-\frac{884}{885}\right) .
$$

When we have a YES instance, OPT is at least $X$. This implies OPT is also at least $\frac{m}{2}$. The second term on the right-hand side is then at most $\frac{1}{2}\left(\alpha-\frac{884}{885}\right) O P T$, so the right-hand side is at least $\frac{\alpha+884 / 885}{2} O P T$.

However, no reserves $\mathbf{r}^{t}$ can get more total revenue on all valuation profiles than OPT. The lefthand side is the expected (over the randomness of $A^{\prime \prime}$ ) average (over the outputs of $A^{\prime \prime}$ to $A^{\prime}$ ) total revenue of $A^{\prime \prime}$ on all valuation profiles. The actual average total revenue lies between 0 and OPT, but we have shown its expectation is at least $\frac{\alpha+884 / 885}{2}$ OPT. By Markov's inequality, this means there is a constant probability that the average total revenue is better than $\frac{884}{885} O P T$, and hence better than $\frac{884}{885} X$. In this event, there must be at least one $\mathbf{r}^{t}$ that results in strictly more than $\frac{884}{885} X$ total revenue on the valuation profiles. The constant success probability only depends on $\alpha$, which was assumed to be a constant. (And, since there is one-sided error, this correctness probability can be amplified to a constant arbitrarily close to 1 by repetitions.)

However, suppose we have a NO instance. This means that OPT is at most $\frac{884}{885} X$, and hence our algorithm cannot find reserves $\mathbf{r}^{t}$ that result in more than $\frac{884}{885} X$ total revenue. This completes the correctness analysis. 


\subsection{General Offline to Online Inapproximability Reduction}

The reduction of Theorem 6.1 can be generalized to a wide class of online problems. Suppose we have an online problem where in every round the algorithm commits to an action and then receives a payoff vector with description length $n$, which can be used to compute the payoff of any action in poly $(n)$ time. Payoffs lie in the range $[0,1]$. The corresponding offline problem is to compute the best fixed action given $m$ (succinct descriptions of) payoff vectors and a probability for each payoff vector occurring. Note that this is a different formulation than our offline problem, which corresponds to the uniform distribution over the $m$ payoff vectors, and with the answer scaled by a factor of $m$. In this formulation, the answer to the problem also lies in the range $[0,1]$. We show that if this offline problem cannot be approximated, then neither can the online problem:

Theorem 6.2. Suppose it is NP-hard to distinguish between offline instances in which the maximum payoff is at least a given parameter $X$, and those instances in which the maximum payoff is at most $\alpha X$, even when $X \geq \beta$ for some constants $\alpha, \beta>0$. Then unless $N P \subseteq R P$, for all constants $\alpha^{\prime}>\alpha$ there is no polynomial-time algorithm for the online problem with no $\alpha^{\prime}$-regret.

Proof. The proof of Theorem 6.1 did not rely on any specific facts about the MMR problem. We can still transform any online algorithm $A$ with $\alpha^{\prime}$-regret $f(T)$ into $A^{\prime}$ with $m$-periodic $\alpha^{\prime}$ regret $f(T / m)$. However, we need to be more detailed with our transformation from $A^{\prime}$ to $A^{\prime \prime}$ to account for the fact that we are no longer using a uniform distribution. We plan to again produce an $A^{\prime \prime}$ that must output the same action for $m$ rounds in a row; these $m$ rounds constitute a block. We augment the problem so the input for each round $t$ in a block comes with a probability $p_{t}$, and the probabilities for a round sum to 1 . These probabilities indicate how much each round is weighted; the (time-averaged) $\alpha$-regret of a weighted online algorithm over a block of valuations $\mathbf{v}^{(k-1) m+1}, \ldots, \mathbf{v}^{k m}$ is:

$$
\alpha \sum_{t=(k-1) m+1}^{k m} p_{t} R\left(\mathbf{r}^{\star}, \mathbf{v}^{t}\right)-\mathbb{E}\left[\sum_{t=(k-1) m+1}^{k m} p_{t} R\left(\mathbf{r}^{(k-1) m+1}, \mathbf{v}^{t}\right)\right],
$$

where $\mathbf{r}^{(k-1) m+1}$ is the action chosen for the entire block. Our $A^{\prime \prime}$ is as before, but instead of choosing a block ordering uniformly at random, we choose round $t$ to be the first in the block with probability $p_{t}$. Again, reordering does not affect the best fixed reserves. However, under this reordering and the definition of $m$-periodic regret for $A^{\prime}$, we get that the $\alpha^{\prime}$-regret of $A^{\prime \prime}$ is $f(T / m)$.

Choosing the smallest $T$ (the number of blocks or equivalently, random samples) such that $f(T)$ is at most $\frac{1}{2} \beta\left(\alpha^{\prime}-\alpha\right)$ requires $T$ to only be poly $(n)$. This choice of $T$ guarantees the expected average payoff of an output of $A^{\prime \prime}$ is at least $\alpha^{\prime} \cdot$ OPT minus $\frac{1}{2} \beta m\left(\alpha^{\prime}-\alpha\right)$. For YES instances, this shows the expected average total payoff is at least $\frac{\alpha^{\prime}+\alpha}{2} X$, so with constant probability some action returned by $A^{\prime \prime}$ will have total payoff more than $\alpha X$. For NO instances, all actions have total payoff at most $\alpha X$. This RP algorithm would solve an NP-hard problem, contradicting our hypothesis. Hence, no such online algorithm can exist.

\section{OPEN QUESTIONS}

Our work suggests several directions for further research.

(1) Is there an approximation algorithm with approximation ratio better than $\frac{1}{2}$ for the MMR problem, for a single-item auction or more generally?

(2) Is there a constant-factor approximation algorithm for the MMR problem in downwardclosed non-matroid environments? 
(3) How generally can an offline approximation algorithm be turned into an equally good computationally efficient online learning algorithm? (See Dudik et al. (2017) for recent progress.)

(4) Is there a good approximation algorithm that is also "stable," meaning that its output does not change much with the addition or deletion of a small number of valuation profiles? A positive result could have implications for both learning (Bousquet and Elisseeff 2002) and privacy (Wang et al. 2016).

\section{ACKNOWLEDGMENTS}

We thank the EC and TEAC reviewers for numerous helpful comments.

\section{REFERENCES}

Saeed Alaei, Jason D. Hartline, Rad Niazadeh, Emmanouil Pountourakis, and Yang Yuan. 2015. Optimal auction vs. anonymous pricing. In Proceedings of the 56th Symposium on Foundations of Computer Science (FOCS'15). 1446-1463.

Martin Anthony and Peter L. Bartlett. 1999. Neural Network Learning: Theoretical Foundations. Cambridge University Press, NY, NY.

Susan Athey and Denis Nekipelov. 2010. A structural model of sponsored search advertising auctions. In Proceedings of the 6th Ad Auctions Workshop, Vol. 15.

Avrim Blum and Jason D. Hartline. 2005. Near-optimal online auctions. In Proceedings of the 16th ACM-SIAM Symposium on Discrete Algorithms (SODA'05). Society for Industrial and Applied Mathematics, 1156-1163.

Olivier Bousquet and André Elisseeff. 2002. Stablity and generalization. F. Mach. Learn. Research 2 (2002), 499-526.

Nicolò Cesa-Bianchi, Claudio Gentile, and Yishay Mansour. 2013. Regret minimization for reserve prices in second-price auctions. In Proceedings of the 24th ACM-SIAM Symposium on Discrete Algorithms (SODA'13). SIAM, New York, NY, $1190-1204$

Nicolò Cesa-Bianchi and Gábor Lugosi. 2006. Prediction, Learning, and Games. Cambridge University Press.

Shuchi Chawla, Hu Fu, and Anna R. Karlin. 2014. Approximate revenue maximization in interdependent value settings. In Proceedings of the 15th ACM Conference on Electronic Commerce (EC'14). 277-294.

Shuchi Chawla, Jason D. Hartline, and Robert Kleinberg. 2007. Algorithmic pricing via virtual valuations. In Proceedings of the 8th ACM Conference on Electronic Commerce (EC'07). 243-251.

Miroslav Chlebik and Janka Chlebikova. 2008. Approximation hardness of dominating set problems in bounded degree graphs. Inform. Comput. 206, 11 (2008), 1264-1275.

Richard Cole and Time Roughgarden. 2014. The sample complexity of revenue maximization. In Proceedings of the 46th ACM Symposium on Theory of Computing (STOC'14). 243-252.

Peter Cramton, Hector Lopez, David Malec, and Pacharasut Sujarittanonta. 2015. Design of the Reverse Auction in the Broadcast Incentive Auction. (2015). An expert report in response to Comment Public Notice FCC 14-191. http://www.cramton. umd.edu/papers2015-2019/cramton-reverse-auction-design-fcc-comment-pn.pdf.

Constantinos Daskalakis and Vasilis Syrgkanis. 2016. Learning in auctions: Regret is hard, envy is easy. In Proceedings of the 57th Symposium on Foundations of Computer Science (FOCS'16). 219-228.

Nikhil R. Devanur, Zhiyi Huang, and Christos-Alexandros Psomas. 2016. The sample complexity of auctions with side information. In Proceedings of the 48th ACM Symposium on Theory of Computing (STOC'16). 426-439.

Peerapong Dhangwatnotai, Tim Roughgarden, and Qiqi Yan. 2015. Revenue maximization with a single sample. Games Econ. Behav. 91 (2015), 318-333.

Shahar Dobzinski, Hu Fu, and Robert Kleinberg. 2011. Optimal auctions with correlated bidders are easy. In Proceedings of the 43rd ACM Symposium on Theory of Computing (STOC'11). 129-138.

Miroslav Dudik, Nika Haghtalab, Haipeng Luo, Robert E. Schapire, Vasilis Syrgkanis, and Jennifer Wortman Vaughan. 2017. Oracle-efficient learning and auction design. In Proceedings of the 58th Symposium on Foundations of Computer Science (FOCS'17). 528-539.

Edith Elkind. 2007. Designing and learning optimal finite support auctions. In Proceedings of the 18th ACM-SIAM Symposium on Discrete Algorithms (SODA'07). SIAM, 736-745.

Yannai A. Gonczarowski and Noam Nisan. 2017. Efficient empirical revenue maximization in single-parameter auction environments. In Proceedings of the 49th ACM Symposium on Theory of Computing (STOC'17). 856-858.

Venkatesan Guruswami, Jason D. Hartline, Anna R. Karlin, David Kempe, Claire Kenyon, and Frank McSherry. 2005. On profit-maximizing envy-free pricing. In Proceedings of the 16th ACM-SIAM Symposium on Discrete Algorithms (SODA'05). SIAM, 1164-1173.

James Hannan. 1957. Approximation to Bayes risk in repeated play. Contrib. Theor. Games 3, 97-139 (1957), 2. 
Jason D. Hartline and Tim Roughgarden. 2009. Simple versus optimal mechanisms. In Proceedings of the 10th ACM Conference on Electronic Commerce (EC'09). 225-234.

Zhiyi Huang, Yishay Mansour, and Tim Roughgarden. 2018. Making the most of your samples. SIAM J. Comput. 47, 3 (2018), 651-674.

Sham M. Kakade, Adam Tauman Kalai, and Katrina Ligett. 2009. Playing games with approximation algorithms. SIAM f. Comput. 39, 3 (2009), 1088-1106.

Adam Kalai and Santosh Vempala. 2003. Efficient algorithms for online decision problems. In Learning Theory and Kernel Machines. Springer, 26-40.

Robert D. Kleinberg and Frank Thomson Leighton. 2003. The value of knowing a demand curve: Bounds on regret for online posted-price auctions. In Proceedings of the 44th Symposium on Foundations of Computer Science (FOCS'03). IEEE Computer Society, New York, NY, 594-605.

Renato Paes Leme, Martin Pal, and Sergei Vassilvitskii. 2016. A field guide to personalized reserve prices. In Proceedings of the 25th International Conference on World Wide Web (WWW'16). International World Wide Web Conferences Steering Committee, 1093-1102.

Andres Munoz Medina and Mehryar Mohri. 2014. Learning theory and algorithms for revenue optimization in second price auctions with reserve. In Proceedings of the 31st International Conference on Machine Learning (ICML'14). 262-270.

Jamie H. Morgenstern and Tim Roughgarden. 2015. On the pseudo-dimension of nearly optimal auctions. In Proceedings of the 29th Conference on Neural Information Processing Systems (NeurIPS'15). 136-144.

Roger B. Myerson. 1981. Optimal auction design. Math. Oper. Res. 6, 1 (1981), 58-73.

Christos Papadimitriou and Mihalis Yannakakis. 1988. Optimization, approximation, and complexity classes. In Proceedings of the 20th ACM Symposium on Theory of Computing (STOC'88). ACM, 229-234.

Amir Ronen. 2001. On approximating optimal auctions. In Proceedings of the 3rd ACM Conference on Electronic Commerce (EC'01). ACM, 11-17.

Tim Roughgarden and Joshua R. Wang. 2016. Minimizing regret with multiple reserves. In Proceedings of the ACM Conference on Economics and Computation (EC'16). ACM, 601-616.

Alexander Schrijver. 2003. Combinatorial Optimization: Polyhedra and Efficiency. Vol. 24. Springer Science \& Business Media.

Kunal Talwar. 2003. The price of truth: Frugality in truthful mechanisms. In Proceedings of the 20th International Symposium on Theoretical Aspects of Computer Science (STACS'03). Springer, 608-619.

Yu-Xiang Wang, Jing Lei, and Stephen E. Fienberg. 2016. Learning with differential privacy: Stability, learnability and the sufficiency and necessity of ERM principle. f. Mach. Learn. Res. 17 (2016), 1-40.

Received December 2016; revised August 2018; accepted July 2019 